\title{
A Modified Algorithm for Reducing Calculation Errors in Large Strain
}

\section{Measurement with Strain Gauges}

\author{
G. Chen ${ }^{1, a} Y$. Deng ${ }^{1,2 b}$ L. Sun ${ }^{3, c}$, T. $X u^{4, d}$
}

${ }^{1}$ East China University of Science and Technology,School of Mechanical and Power Engineering, No.130 Meilong Road,Shanghai 200237, China.

${ }^{2}$ Hubei Special Equipment Safety Inspection and Research Institute ,No.352 Xudong street,Wuhan 430077, China

${ }^{3}$ China Special Equipment Inspection and Research Institute, 2 Building,Xiyuan,Hepingjie Beijing 100013,China

aemail: chen_gang@sac.gov.cn, bemail:deng_ych@126.com

cemail: sunliang@csei.org.cn, demail: audreytxu@126.com

Keywords: large strain, error, algorithms, measurement

\begin{abstract}
There is no simple linear relationship between strain and potential in strain measurement with strain gauges, especially for large strain measurements. In this paper, a modified algorithm was proposed to improve the accuracy of strain obtained from measured voltage. The strain was calculated from a nonlinear relationship between voltage and strain rather than a linear simplification. Moreover, the corrections for different sensitivity factors of strain gauges and lead wire resistance were considered. The proposed method was suitable for both large and small strain measurements using a quarter bridge, and validated by experimental tests. It is also very easy to be implemented as a software form and used in scientific tests and engineering applications.
\end{abstract}

\section{Introduction}

The strain gauges have widely been used for the small strain measurement in scientific research and engineering applications due to their high precision, low cost and convenience. When large strain measurements are required, optical measurement methods are commonly used. However, on some occasions, for example, for the large strain measurement in a burst test of pressure vessel, strain gauge technology is more suitable because of the high risk and damages.

There are three important things should be considered in using strain gauge technology to measure a large strain[1]. The first is the linearity of strain gauge and adhesion quality. For a small strain measurement, it is a mature technique. Tokyo Measure Institute and other companies have made strain gauge and adhesion suitable for large strain measurement. The second is the linearity of instrument voltage. The linearity within the range of large voltage can be guaranteed by adjusting the amplifier. The third is the relationship between voltage and strain value from the strain gauges. For a small strain measurement, a linear proportional relationship is suitable; for a large strain measurement, using a linear relationship will cause too large an error. In this study, a modified method will be developed to build a nonlinear relationship between the voltage and strain to reduce measurement error. 


\section{Basic principle of Strain Gauge Technology}

The relationship between strain and resistance change

The relationship between strain and resistance change can be expressed as

$$
k=\frac{\Delta R}{R} / \varepsilon
$$

Where $k$ is the sensitivity coefficient of strain gauge, provided by the manufacturer. From Eq. (1), the strain in a structure can be measured by measuring the resistance change of the strain gauge attached to the structure.

\section{Bridge Circuit}

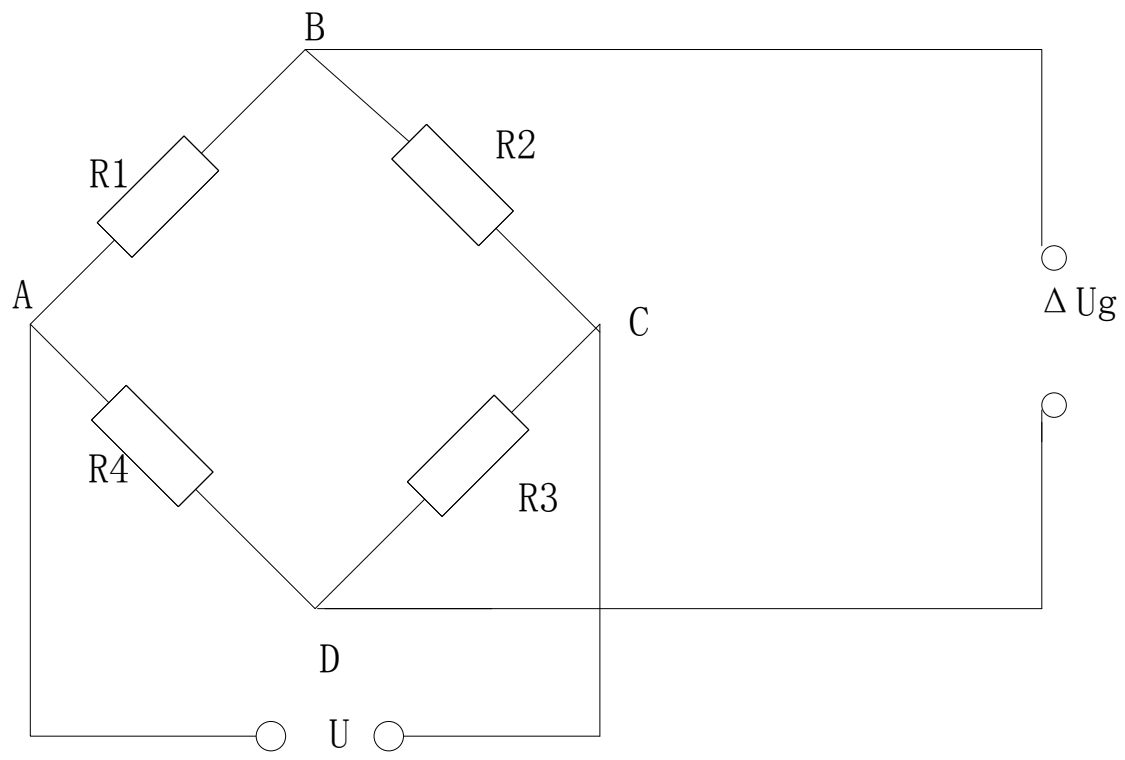

Figure 1 Wheatstone bridge circuit

Figure 1 shows a Wheatstone bridge circuit. The bridge output voltage between point $\mathrm{B}$ and point $\mathrm{D}$ is:

$$
\Delta U g=\left(\frac{R_{1}}{R_{1}+R_{2}}-\frac{R_{4}}{R_{3}+R_{4}}\right) U
$$

where $U$ is the supply voltage. 


\section{Standard Strain Gauge Simulator}

For a half-bridge circuit, we have

$$
\begin{aligned}
& R_{1}=R+\Delta R \\
& R_{2}=R-\Delta R \\
& R_{3}=R \\
& R_{4}=R
\end{aligned}
$$

Substituting the equations above into Eq.(2) yields

$$
\Delta U_{g}=\frac{U}{2} \cdot \frac{\Delta R}{R}
$$

Which shows a linear relationship between $\Delta U_{g}$ and $\Delta R$. A standard strain gauge instrument was made based on this relationship to measure the strain.

\section{Quarter Measuring Bridge}

In engineering applications, a quarter bridge is widely used. We have

$$
\begin{aligned}
& R_{1}=R+\Delta R \\
& R_{2}=R_{3}=R_{4}=R
\end{aligned}
$$

Substituting the equations above into Eq.(2) yields

$$
\Delta U_{g}=\frac{U}{4} \cdot \frac{\Delta R}{R+\frac{1}{2} \cdot \Delta R}
$$

\section{Strain Calculation Method}

\section{Normal Strain Calculation Method}

Since $\Delta R / R$ is very small, Equation (4) can be approximately expressed as

$$
\Delta U_{g}=\frac{U}{4} \cdot \frac{\Delta R}{R} \cdot\left[1-\frac{1}{2} \cdot \frac{\Delta R}{R}+\left(\frac{1}{2} \cdot \frac{\Delta R}{R}\right)^{2}-\cdots \cdots\right]
$$

For an approximation, Eq. (5) is rewritten as

$$
\begin{aligned}
\Delta U_{g} & =\frac{U}{4} \cdot \frac{\Delta R}{R} \\
& =\frac{U}{4} \cdot k \varepsilon
\end{aligned}
$$

So

$$
\varepsilon=\frac{4}{k} \cdot \frac{\Delta U_{g}}{U}
$$


The approximation error introduced by using Eq.(6) to calculate $\Delta U_{g}$ instead of Eq.(5) is

$$
e=\frac{1}{2} \cdot \frac{\Delta R}{R}=\frac{k \varepsilon}{2}
$$

When $\mathrm{k}=2, \operatorname{Eq}(7)$ is expressed as

$$
e=\varepsilon
$$

Thus, for a strain of $10 \%$, the error introduced by the approximation is $10 \%$. Since Eq. (6) exhibits a linear relationship between the voltage and strain changes and its approximation error is small for small strain measurements, it is widely used.

\section{Modified algorithm of strain calculation}

Eq. (4) shows that the relationship between voltage and strain is not linear. In practical applications of large strain measurements, a relationship curve between voltage and strain is calibrated by experiments in order to reduce the calculation error in [2]. This method is not convenient and not adaptable. Therefore, it is more suitable to directly use Eq. (4) rather than the simplified linear relationship between voltage and strain.

From Equation (4), the resistance change rate can be expressed as

$$
\frac{\Delta R}{R}=\frac{1}{\frac{1}{4} \cdot \frac{U}{\Delta U_{g}}-\frac{1}{2}}
$$

which can be used to calculate the strain.

\section{Correction of measurement Parameters}

\section{Temperature Compensation}

In a half bridge, two strain gauges are used: one is used to measure strain, the other is for temperature compensation. The resistance can be expressed as

$$
\begin{aligned}
& R_{1}=R+\Delta R+\Delta R_{t} \\
& R_{2}=R+\Delta R_{t} \\
& R_{3}=R_{4}=R
\end{aligned}
$$

Substituting the resistances into Eq. (3) yields

$$
\Delta U_{g}=\frac{U}{4} \cdot \frac{\Delta R}{R} \cdot \frac{1}{1+\frac{1}{2} \cdot \frac{\Delta R}{R}+\frac{\Delta R_{t}}{R}}
$$

In the room temperature $\Delta R_{t}=0$, Eqs. (10) and (4) are equivalent.

\section{Sensitivity Correction of Strain Gauge}

The sensitivity factor of instrument is different from that of strain gauges, which can be expressed as:

$$
\frac{\Delta R}{R}=k_{\mathrm{in}} \cdot \varepsilon_{\mathrm{in}}
$$

Where $k_{\text {in }}$ is the sensitivity factor of instrument, $\varepsilon_{i n}$ is the measurement of instrument. 
Thus the strain value from the strain gauge can be written as:

$$
\varepsilon=\frac{1}{k} \cdot \frac{\Delta R}{R}=\frac{k_{\mathrm{in}}}{k} \cdot \varepsilon_{\mathrm{in}}
$$

Equation (11) can be used to correct the sensitivity factor of different strain gauges to be applicable for the same strain measurement instrument.

\section{Lead wire Resistance Attenuation Correction}

The error due to lead wire resistance attenuation can be corrected by using a three-wire circuit, as shown in Figure 2, where strain gauges $R_{1}$ and $R_{2}$ are used for strain measurement and temperature compensation, respectively. The strain with a lead wire resistance correction is written as

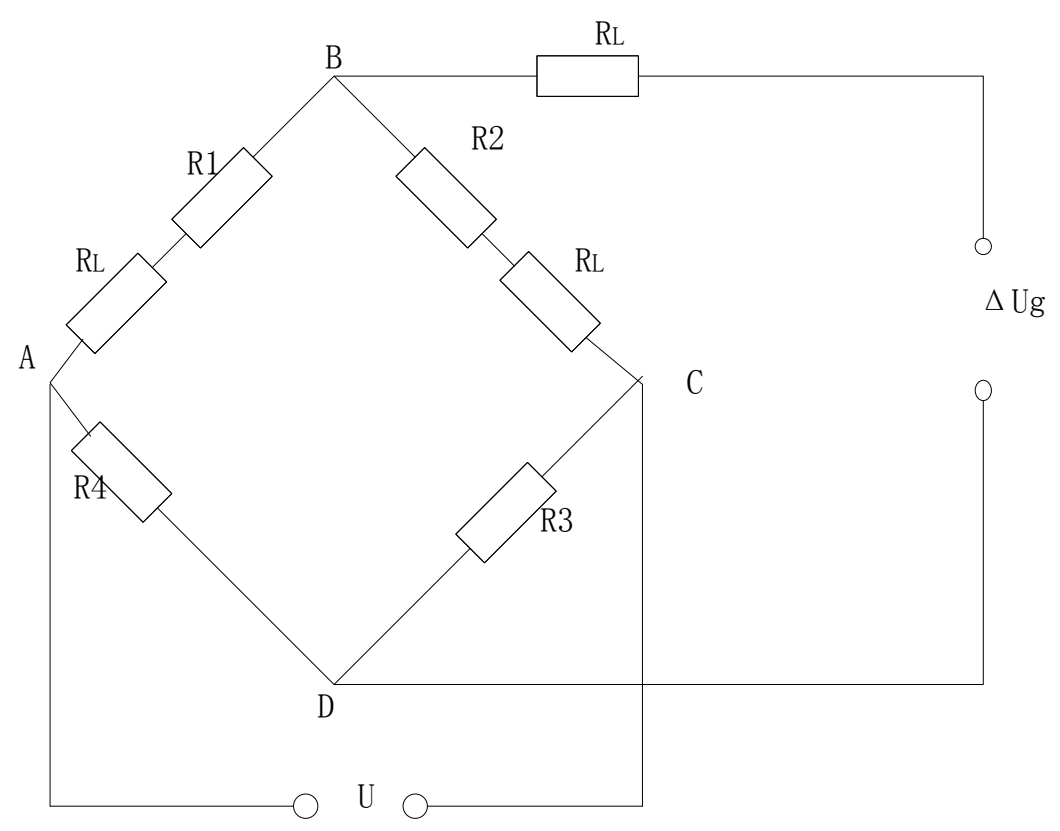

Figure 2 A Three-Wire Circuit diagram

$$
\begin{aligned}
& R_{1}=R+\Delta R+R_{L} \\
& R_{2}=R+R_{L} \\
& R_{3}=R_{4}=R
\end{aligned}
$$

We can get

$$
\varepsilon=\frac{R}{R+R_{L}} \cdot \varepsilon_{\mathrm{in}}
$$




\section{Strain Measurements with corrections}

The strain measurement with temperature compensation and lead wire resistance correction can be obtained by combining Eqs.(11) and (12), expressed as:

$$
\varepsilon=\frac{k_{\mathrm{in}}}{k} \cdot \frac{R+R_{L}}{R} \cdot \varepsilon_{\mathrm{in}} \ldots \ldots
$$

Set $C=\frac{k}{k_{\mathrm{in}}} \cdot \frac{R+R_{L}}{R}$ which presents the corrections of temperature compensation and lead wire resistance corrections, the true strain can be expressed as:

$$
\varepsilon=C \cdot \varepsilon_{\mathrm{in}}
$$

where $\varepsilon_{\text {in }} \propto \Delta U_{g}^{\text {in }}$. The voltage change can be expressed as:

$$
\Delta U_{g}=C \cdot \Delta U_{g}^{\mathrm{in}}
$$

Substituting Eq. (15) into Eq. (9) yields

$$
\frac{\Delta R}{R}=\frac{1}{\frac{1}{4} \cdot \frac{U}{C \cdot \Delta U_{g}^{\mathrm{in}}}-\frac{1}{2}}
$$

Then substituting Eq. (16) into Eq.(1) yields

$$
\varepsilon=\frac{1}{k} \cdot \frac{\Delta R}{R}=\frac{1}{k} \cdot \frac{1}{\frac{1}{4} \cdot \frac{U}{C \cdot \Delta U_{g}^{\mathrm{in}}}-\frac{1}{2}}
$$

Where $k$ is the sensitivity factor of strain gauges, $k_{i n}$ is the sensitivity factor of instrument, $C=\frac{k}{k_{\mathrm{in}}} \cdot \frac{R+R_{L}}{R}$.

\section{Experimental Validation}

\section{Experimental Instruments}

A dynamic strain gauge instrument, SDY 21202D, developed by Beidaihe Experimental Electric Institution and the author, was used to validate the proposed method. The measure range of strain is $0-320000 \mu \varepsilon$. The developed instrument was calibrated by using a standard strain gauge instrument ,BYM-6, made by Beidaihe Experimental Electric Institution, a digital multimeter,HP34401A ( 6 1/2 byte), made by Hewlett-Packard, and a signal generator,TD1017, made by Tianjin Zhonghuan Electronic Instrument Company. The maximum instrumental linear error of SDY21202D is $0.01 \%$. Other instruments used included a standard strain gauge simulator,SDY2301,made by Beidaihe Experimental Electric Institution, with a sensitivity factor of 
2 and measurement ranges are 200000, 1000000,1500000,200000,250000 and $300000 \mu \varepsilon$, and a standard strain gauge simulator, SDY2306, made by Beidaihe Experimental Electric Institution, with a sensitivity of 2 and a measure range from $0.1-100000 \mu \varepsilon$ and the maximum error of SDY2301 and SDY2306 is $0.05 \%$.

\section{Experimental Procedure}

Strain gauge simulators, SDY 2301 and SDY2306, were used to simulate the behavior of strain. Quarter measuring bridge was used to response the signal from strain gauge simulators, and SDY2102D dynamical strain gauge instrument was used to measure the strain. Quarter measuring bridge could be calculated according to three-wire circuit method as $R_{L}$ is zero.

\section{Experimental Results}

Table 1 shows that the calculation errors of strains obtained from both simplified equation (Eq.(6)) and modified equation (Eq.(17)) are small for a small strain measurement. The error is always small when Eq. (17) is used. However, the error increases with the increasing strain value when Eq. (6) is used: the error increases to more than $1.16 \%$ when the strain value reaches $10000 \mu \varepsilon$, which can not be neglected; the error increases to $9.12 \%$ when the strain value reaches $100000 \mu \varepsilon$, which is not acceptable.

We also did another group experiment by three-wire circuit method and got the same law as Table 1. Lead-wire long is 50 meters and per lead-wire dimension is $0.5 \mathrm{~mm}$, we measure lead-wire resistance $R_{L}$ is $2 \Omega, R$ chooses $120 \Omega$.

\section{Conclusion}

In this paper, a modified method was proposed to calculate the strain from the resistance change of the strain gauge, which is suitable for both small and large strain measurements. The corrections for temperature compensation and lead wire resistance attenuation were also considered. The developed method can improve the accuracy of strain measurements and can be used in the scientific research and engineering applications. 
Table 1 Strain Results Measured from quarter measuring bridge

\begin{tabular}{|c|c|c|c|c|c|c|}
\hline \multirow[t]{2}{*}{$\begin{array}{l}\text { Strain } \\
\text { values } \\
(\mu \varepsilon)\end{array}$} & \multicolumn{2}{|c|}{$\begin{array}{l}\text { Measuring Voltage of } \\
\Delta U_{g}^{\text {in }}\end{array}$} & \multicolumn{2}{|c|}{$\begin{array}{ll}\text { Strain } & \text { value } \\
\text { calculated } & \text { using } \\
\text { Equation(6) } & \end{array}$} & \multicolumn{2}{|c|}{$\begin{array}{l}\text { Strain value calculated } \\
\text { using Equation }(17)\end{array}$} \\
\hline & $\begin{array}{l}\Delta U_{g}^{i n} \text { after } \\
\text { enlargement } \\
\text { by amplifier } \\
\text { (V) }\end{array}$ & $\begin{array}{l}\text { Enlargement } \\
\text { Factor by } \\
\text { amplifier for } \\
\text { measuring } \\
\Delta U_{g}^{i n}\end{array}$ & $\begin{array}{l}\text { Strain } \\
\text { Value } \\
(\mu \varepsilon)\end{array}$ & Error (\%) & $\begin{array}{l}\text { Strain } \\
\text { Value } \\
(\mu \varepsilon)\end{array}$ & Error $(\%)$ \\
\hline 500 & 0.124 & \multirow[t]{11}{*}{250} & 500 & 0 & 500.2501 & 0.050025 \\
\hline 1000 & 0.249 & & 996 & -0.400 & 996.993 & -0.3007 \\
\hline 1500 & 0.374 & & 1496 & -0.267 & 1498.241 & -0.11724 \\
\hline 2000 & 0.498 & & 1992 & -0.400 & 1995.976 & -0.2012 \\
\hline 2500 & 0.623 & & 2492 & -0.320 & 2498.226 & -0.07098 \\
\hline 3000 & 0.747 & & 2988 & -0.400 & 2996.955 & -0.1015 \\
\hline 3500 & 0.871 & & 3484 & -0.457 & 3496.181 & -0.10912 \\
\hline 4000 & 0.995 & & 3980 & -0.500 & 3995.904 & -0.10241 \\
\hline 4500 & 1.119 & & 4476 & -0.533 & 4496.125 & -0.08612 \\
\hline 5000 & 1.241 & & 4964 & -0.720 & 4988.764 & -0.22472 \\
\hline 10000 & 2.471 & & 9884 & -1.160 & 9982.669 & -0.17331 \\
\hline 15000 & 0.369 & \multirow[t]{12}{*}{25} & 14760 & -1.600 & 14981.12 & -0.12586 \\
\hline 20000 & 0.490 & & 19600 & -2.000 & 19991.84 & -0.0408 \\
\hline 25000 & 0.609 & & 24360 & -2.560 & 24968.23 & -0.1271 \\
\hline 30000 & 0.727 & & 29080 & -3.067 & 29950.97 & -0.16342 \\
\hline 35000 & 0.844 & & 33760 & -3.543 & 34939.56 & -0.17269 \\
\hline 40000 & 0.960 & & 38400 & -4.000 & 39933.44 & -0.16639 \\
\hline 45000 & 1.075 & & 43000 & -4.444 & 44932.08 & -0.15093 \\
\hline 50000 & 1.192 & & 47680 & -4.640 & 50067.2 & 0.134409 \\
\hline 75000 & 1.745 & & 69800 & -6.933 & 75037.63 & 0.050168 \\
\hline 100000 & 2.272 & & 90880 & -9.120 & 99964.8 & -0.0352 \\
\hline 125000 & 2.777 & & 111080 & -11.1360 & 124960.6 & -0.0315 \\
\hline 150000 & 3.260 & & 130400 & -13.067 & 149954 & -0.03067 \\
\hline
\end{tabular}

\section{Acknowledgement:}

This paper is sponsored by the 11th Five-year China National Key Technology R\&D Program,No.2006BAK02B02.

\section{References}

[1] Zhang Ruyi, Shen Guangling, Li Caodi, Strain Measurement and Sensor, Tsinghua University Press, Beijing P.R.China, Jan. 1999.

[2] Chen Jian, Kang Xuejun, The High Elongation Strain Gages (20\%) for Measuring Large Plastic Deformation, (Chinese) Journal of Experimental Mechanics, Vol.9 No.2, June. 1994,P175-180. 